\title{
BMJ Open The impact of decision aids in patients with colorectal cancer: a systematic review
}

\author{
Jenaya Goldwag, ${ }^{1,2}$ Priscilla Marsicovetere, ${ }^{2,3}$ Peter Scalia, ${ }^{\oplus 4}$ \\ Heather A Johnson, ${ }^{2,5}$ Marie-Anne Durand, ${ }^{4}$ Glyn Elwyn, ${ }^{\oplus}$ Srinivas J Ivatury ${ }^{1,2}$
}

To cite: Goldwag J,

Marsicovetere P, Scalia P, et al.

The impact of decision aids in patients with colorectal cancer: a systematic review. BMJ Open 2019;9:e028379. doi:10.1136/ bmjopen-2018-028379

- Prepublication history and additional material for this paper are available online. To view these files, please visit the journal online (http://dx.doi. org/10.1136/bmjopen-2018028379).

Received 05 December 2018 Revised 16 August 2019 Accepted 28 August 2019

Check for updates

(C) Author(s) (or their employer(s)) 2019. Re-use permitted under CC BY-NC. No commercial re-use. See rights and permissions. Published by BMJ.

${ }^{1}$ Department of Surgery, Dartmouth Hitchcock Medical Center, Lebanon, New Hampshire, USA

${ }^{2}$ Clinical Education, Geisel School of Medicine, Hanover, New Hampshire, USA

${ }^{3}$ Master of Physician Assistant Studies Program, Franklin Pierce University, West Lebanon, New Hampshire, USA

${ }^{4}$ The Dartmouth Institute for Health Policy and Clinical Practice, Dartmouth College, Lebanon, New Hampshire, USA

${ }^{5}$ Biomedical Libraries,

Dartmouth College, Hanover,

New Hampshire, USA

Correspondence to

Dr Srinivas J Ivatury;

Srinivas.J.Ivatury@hitchcock. org

\section{ABSTRACT}

Objectives Our aim was to conduct a systematic review of the literature to determine the impact of patient decision aids (PDA) on patients facing treatment decisions for colorectal cancer.

Design Systematic review.

Data sources Sources included Embase, Medline, Web of Science, CINAHL and the Cochrane Library from inception to June, 20, 2019.

Eligibility criteria We included randomised controlled trials (RCTs), cohort studies, mixed methods and case series in which a PDA for colorectal cancer treatment was used. Qualitative studies were excluded from our review. Data extraction and synthesis Following execution of the search strategy by a medical librarian, two blinded independent reviewers identified articles for inclusion. Two blinded reviewers were also responsible for data extraction, risk of bias and study quality assessments. Any conflict in article inclusion or extraction was resolved by discussion.

Results Out of 3773 articles identified, three met our inclusion criteria: one RCT, one before-and-after study and one mixed-method study. In these studies, the use of a PDA for colorectal cancer treatment was associated with increased patient knowledge, satisfaction and preparation for making a decision. On quality assessment, two of three studies were judged to be of low quality.

Conclusion A paucity of evidence exists on the effect of PDA for colorectal cancer treatment with existing evidence being largely of low quality. Further investigation is required to determine the effect of decision aids for colorectal cancer treatment as well as reasons for the lack of PDA development and implementation in this area. Prospero registration number CRD42018095153.

\section{INTRODUCTION}

Treatment decisions for colorectal cancer can be complex and multimodal, with significant variability and controversy. Patients diagnosed with colorectal cancer have many options for treatment including chemotherapy, surgery and radiation therapy depending on their cancer stage, medical history and preferences. For some clinical situations, such as stage II colon cancer, there is significant variability between options, including surgery alone versus surgery and chemotherapy, as well as

\section{Strengths and limitations of this study}

A broad search strategy as well as a firm adherence to systematic review methodology make this a comprehensive review on decision aids used for colorectal cancer treatment.

- A risk of bias tool and/or a quality assessment tool was used to assess randomised controlled trials or non-randomised trials respectively.

- Including a broad number of outcomes in the inclusion criteria limits the ability to make discrete conclusions.

- There were not enough articles identified to perform a meta-analysis.

the choice of chemotherapy. ${ }^{1}{ }^{2}$ In addition, patients diagnosed with rectal cancer often have to decide between two equally efficacious, but lifestyle altering, surgical options: bowel reconnection with low anterior resection (LAR) versus permanent colostomy with abdominal perineal resection (APR). Further, additional factors exist for many patients increasing decision complexity including the presence or absence of additional colon polyps, concomitant cancers and genetic predisposition. These preference-sensitive decisions can be overwhelming to patients and their families and there can be substantial variation in treatment preferences. ${ }^{34}$

In general, many cancer patients prefer to be actively and collaboratively involved in disease-related decisions. ${ }^{5-8}$ As these decisions can be challenging for patients, often occurring at an emotional time, patient decision aids (PDA) have been developed to provide evidence-based information on treatment options and help patients clarify and communicate the personal values they associate with different options for treatment. ${ }^{9}{ }^{10} \mathrm{PDA}$ are evidence-based tools designed to help patients make informed choices by providing information on the pros, cons, risks, probabilities and scientific uncertainty of available 
options prior to making a decision. ${ }^{11} 12$ PDA can be used when there are multiple reasonable options, when no single option has a clear advantage over the others in terms of health outcomes, or when each option has benefits and harms that patients value differently. ${ }^{13}$ PDA have been shown to increase patient knowledge, reduce decisional conflict, help patients make appropriate decisions and can have a positive effect on patient-clinician communication. ${ }^{13}$

PDA have been successful in helping patients make treatment decisions in breast, prostate and lung cancerother cancer types with similar treatment complexity to colorectal cancer. ${ }^{14-16}$ The impact of PDA in the treatment of colorectal cancer, however, is unclear. Most PDA research regarding colorectal cancer has focused on screening options for prevention as opposed to treatment decisions after diagnosis. ${ }^{17}$ As patients diagnosed with colorectal cancer must also make complex preference sensitive decisions about treatment, we aimed to systematically evaluate the effect of PDA on outcomes associated with colorectal cancer treatment and clinical practice.

\section{METHODS}

\section{Protocol and registration}

We conducted a systematic review, reported in this review using the PRISMA guidelines, of studies that used a colorectal cancer treatment patient decision aid as the intervention. Prior to beginning our search, we published our study protocol in PROSPERO (registration \# CRD42018095153)

\section{Patient and public involvement statement}

The study was performed in hopes to broaden knowledge about PDA for treatment decisions in colorectal cancer care. No patients participated in design or production of this systematic review. In particular, no patients were involved in the development of the research question or outcomes measures, recruitment or conduct, or other aspects of the review.

\section{Eligibility criteria}

We used the Population, Intervention, Comparison, Outcome and Study design criteria to determine eligibility. To be included, studies had to be randomised controlled trials (RCTs), non-randomised controlled trials (NRCT), retrospective or prospective cohort studies, mixed methods, or case series. Any purely qualitative studies or case reports were excluded. Our population did not contain any age restrictions, and included patients diagnosed with colon or rectal cancer needing to decide between two or more management options for treatment. The intervention is a PDA which is a tool designed to inform patients about treatment options and to facilitate patient participation in decision making. ${ }^{112}$ The decision aids could be in any format and used at any time or location, before, during, or after a clinical encounter. The control group would be standard counselling, non-decision aids, or no control group if applicable. We included all study-specified primary and secondary outcomes that related to patients use of the decision aid such as, knowledge gained from PDA, usability of PDA, patient satisfaction of PDA etc. We excluded articles focusing on decision aids or risk calculators that were used only by physicians to guide management of colorectal cancer treatment or implementation of decision aids.

\section{Information sources and search}

With assistance from our medical librarian (HAJ), we developed an electronic search strategy for the following databases: Embase, Medline, Web of Science, CINAHL and the Cochrane Library from inception to June 17, 2019 (please see online supplementary appendix 1 for a summary of the search results). We also looked at conference proceedings from the American Society of Colorectal Surgery annual meeting 2010-2019. We identified articles that assessed decision aids in patients with colorectal cancer, employing text words and database-specific subject headings (eg, MeSH,) such as 'colon cancer', 'rectal cancer', 'decision aids' and 'decision making'. For the purposes of the search, we did not impose any restrictions on language, publication type, or publication date. In addition, we performed a citation search using the 'cited by' option on Google Scholar and 'related searches' on PubMed. We manually checked references for all articles identified as meeting our eligibility requirements for added sensitivity. See online supplementary appendix 2 for search terms used for each database.

\section{Study selection}

We used Rayyan, a systematic review web application, to help facilitate the screening process. ${ }^{18}$ The articles were listed alphabetically so that two reviewers (SJI, HAJ), blinded to each other's results, could independently review articles with first author last names between A-L and two additional similarly blinded reviewers (JG, PM) could independently review articles with first author last names between M-Z. During this initial screening, titles and abstracts were reviewed. Disagreements about inclusion were resolved by discussion by the involved reviewers. If necessary, a third reviewer either (JG or SJI) also contributed to the discussion. After completing the initial screening, two reviewers (SJI, JG) reviewed the full text of the remaining articles. Any conflicts about eligibility at this time were also resolved by discussion.

\section{Data collection process}

\section{For randomised controlled trials}

The data extraction sheet, piloted prior to use, included the following information: study author, publication year, publication type, country, study aims, description of participants (age, gender, education levels, etc.), intervention (what type of DA, when implemented, timing etc.), control group, primary outcome and secondary outcome if applicable. Two reviewers independently extracted 
the data from the included articles. Disagreements were resolved by discussion.

\section{For non-randomised studies}

The data extraction sheet and data extraction methods for non-randomised studies was identical to that for the RCT.

\section{Risk of bias (RCT) and quality assessment (NRCT)}

Risk of bias for RCTs

The risk of bias was assessed by two independent reviewers (SJI, JG) using the Cochrane Collaborations Risk of Bias Tool. ${ }^{19}$ This tool is used to evaluate RCTs in seven domains to judge whether each domain is of high, low, or unclear risk of bias. Disagreements were resolved by discussion.

\section{Quality assessment for NRCTs}

The Downs and Black Checklist was used by two independent reviewers (SJI, JG) to assess the quality of the non-randomised studies. ${ }^{20}$ The reviewer assesses five domains (reporting, external validity, bias, confounding, power) by assigning a 'yes' or 'no' to 27 questions. The answer determines if a point(s) is awarded for that particular question. The highest possible score is 30 with a higher score associated with a higher quality study. This assessment tool was chosen as it has been used previously for pre-post and/or mixed methods studies. ${ }^{21} 22$ Disagreements were resolved by discussion.

\section{RESULTS}

\section{Study characteristics}

A total of 5594 articles were initially identified with 3773 left to review after duplicates were removed. After screening titles and abstracts, 36 articles were left for full review. After assessing the full articles there were three studies ${ }^{23-25}$ included in our final analysis, see figure 1 .

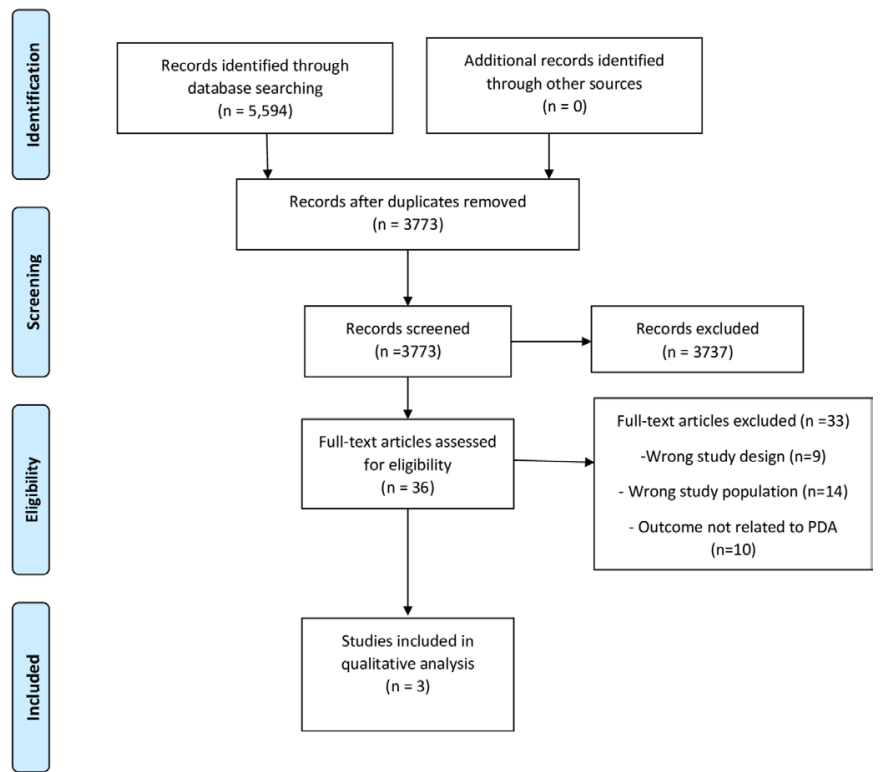

Figure 1 Summary of the review process. PDA, patient decision aids.
This included one RCT, one before-and-after study and one mixed methods study. Characteristics for the three included studies are shown in table 1.

\section{Risk of bias and quality assessment}

Risk of bias: randomised controlled trial

There was a low risk of selection, detection, or attrition bias, with a moderate risk of performance bias found due to inability to blind participants. Reporting bias was felt to be low-moderate because the study was performed in two locations and reported in aggregate. Please see online supplementary appendix 3 for further details to support judgements.

\section{Quality assessment: non-randomised studies}

According to the Downs and Black Checklist, both non-randomised studies were considered to be low quality. The before-and-after study scored 13 out of 30, and the mixed methods study scored 8 out of 30 . In addition, both studies have a significant risk of bias and confounding, due to lack of control group or randomisation.

\section{Study specific results}

Study 1: Leighl et al (Australia, Canada)

This RCT took place in Australia and Canada and included a total of 207 patients, 100 in the control group and 107 in the intervention group. All patients carried a diagnosis of metastatic colorectal cancer and were meeting with an oncologist for the first time to discuss and decide between chemotherapy options. The control group received consultation alone, while the intervention group received consultation plus a decision aid. The decision aid consisted of a paper booklet reviewed during the initial visit on chemotherapy options, as well as a take home booklet and audiotape. The decision aid in this study had been pilot tested and altered based on patient feedback. ${ }^{26}$ Patients completed a series of different questionnaires prior to randomisation and at multiple intervals after the initial consultation. The primary objective of the study was to evaluate patient understanding, via a modified Fiset ${ }^{27}$ and Brundage ${ }^{28}$ questionnaire, and satisfaction with the decision made via the 'satisfaction with decision scale'. ${ }^{29}$ Secondary outcomes included decisional conflict, which evaluated patients' uncertainty with the decision and factors contributing to that uncertainty, assessed via the 'decisional conflict scale' ${ }^{30}$ and readiness to make a decision immediately after consultation. The intervention group had an improved understanding of chemotherapy options 1-2 weeks postconsultation when compared with the control group $(\mathrm{p}<0.001)$, although this is of unclear clinical significance. Patient satisfaction was found to be high and the decisional conflict score was similar in both groups. The Canadian patient population was found to be more likely to feel ready to make a treatment decision immediately after consultation $(86 \% \mathrm{v}$ $42 \%, \mathrm{p}<0.001)$, but had a higher decisional conflict scores $(38 \mathrm{v} 34, \mathrm{p}<0.002)$ when compared with the Australian population. 


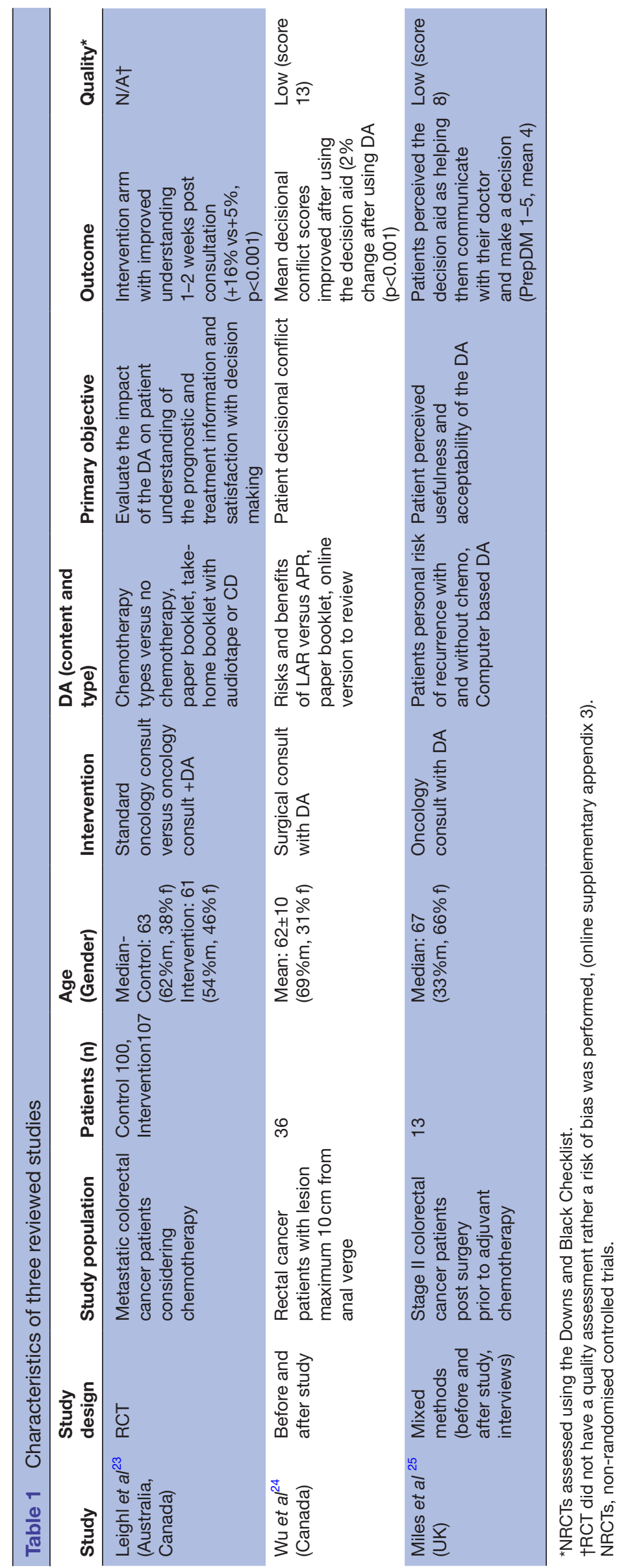

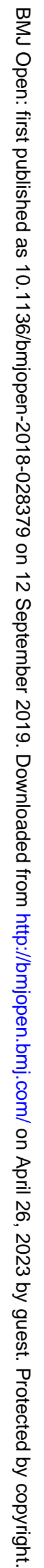


Study 2: Wu et al (Canada)

This before-and-after study took place in Canada and UK. They included a total of 36 patients who were diagnosed with rectal cancer. The study introduced their decision aid during or after consultation with a surgeon to aid in deciding between two surgical options. The decision aid consisted of a paper booklet on the topic of LAR versus APR and sent participants home with a link to an online decision aid. Patients completed questionnaires following initial surgical consultation and after reviewing the decision aid. The primary outcome was decisional conflict. Secondary outcomes included knowledge, choice preference and acceptability of the decision aid. Mean decisional conflict scores were improved by $24.2 \% \quad(p=0.0001)$ after the use of the decision aid. Patient knowledge also increased 37\% $(\mathrm{p}<0.0001)$. The decision aid had variable impact on choice preference, with some patients changing their preference between LAR, APR and neutral after using the DA, with no statistically significant trend toward neutral or either surgical option. In terms of acceptability, $85 \%$ of participants felt the decision aid had good/excellent information about options and 97\% would recommend it to others.

\section{Study 3: Miles et al (United Kingdom)}

This mixed method study took place in Canada and UK. A total of 13 patients diagnosed with stage II colorectal cancer postsurgery prior to chemotherapy were included. They introduced their decision aid during the patient's consultation with an oncologist to help decide which, if any, chemotherapy was right for the patient. The decision aid consisted of a computer-based DA on chemotherapy options and participants were sent home with reference material. Study patients completed a postintervention questionnaire as well as participated in semi-structured interviews. The results of the interviews are not included in this analysis as qualitative research was excluded from this review. The primary outcome was patient-perceived usefulness of the decision aid assessed on the Preparation for Decision Making Scale. The decision aid scored a favourable 4.28 out of five on the Preparation for Decision Making Scale. ${ }^{31}$ Eleven of 12 patients participating ultimately declined chemotherapy.

\section{DISCUSSION}

Our systematic review found limited evidence on the use of PDAs for patients facing treatment decisions for colorectal cancer. We found three articles, two of which were low quality, which evaluated PDA for the treatment of colorectal cancer. These studies found that PDAs improved patient knowledge, facilitated shared decision making, and were well-accepted by patients. However, the results of these studies must be interpreted with caution given the low quality of two of the three articles. Although these studies supported the use of PDAs in this population, there is insufficient evidence to draw definitive conclusions on the impact of PDAs in the treatment of colorectal cancer given the paucity of studies.

Strengths of this review include our engagement with a medical librarian (HAJ) in order to fully review the available literature, and our adherence to the guidelines on how to appropriately conduct and report a systematic review. Potential limitations of our methods include possible omission of studies, although unlikely given our search strategy. Another limitation is the inability to perform subgroup analysis due to the small number of articles identified which are of low quality and have low numbers of participants. There are also limitations to interpretation to the data, such as the heterogeneity of patent participants, as well as the low quality of the two NRCTs. The risk of bias and confounding in these studies make it difficult to delineate clear effects from the target interventions.

This review determined that the current literature evaluating decision aids for colorectal cancer treatment is sparse and of low quality. In addition, the quality of the decision aids themselves is unclear. This gap in the literature is especially noticeable when compared with decision aids developed for treatment of other common cancers such as breast, lung and prostate. ${ }^{14-16}$ Given a similar complexity and variety of treatment options available for colorectal cancer, particularly stage II colon cancer or rectal cancer, it is unknown why there is such a paucity of literature on the use of decision aids in this population. It is possible that an emphasis in preventative care has shifted the research towards colorectal cancer screening since screening rates are lower than other common cancers. ${ }^{17} 32$ Other possible causes include lack of provider comfort and understanding of decision aid benefits and or stigma associated with bowel diseases that may cause investigators less likely to pursue the topic.

Although the evidence in this review to support the use of PDAs for those with colorectal cancer treatment is suboptimal, a recent Cochrane systematic review with over 100 RCTs shows that these interventions improve patient outcomes. ${ }^{13}$ PDAs increase knowledge of the treatment options, risk perception, preparedness to make a decision and can facilitate patient-centred care. ${ }^{13}$ Patients diagnosed with colorectal cancer want to be more involved in the decision-making process and have information needs that are not currently being addressed. ${ }^{33-35}$ In addition, this population has different levels of engagement in the decision making process and has expressed that many treatment decisions, such as chemotherapy and surgical choice, are preference sensitive. ${ }^{3436}$ The need to improve healthcare delivery, and the desire for patients to be involved in the preference-sensitive decision regarding treatment, indicates that PDAs would be beneficial for patients diagnosed with colorectal cancer. Future studies, ideally RCTs, should focus on high quality PDAs to see if they can truly improve knowledge, increase facilitated decision making and are associated with increased patient satisfaction. 


\section{CONCLUSIONS}

There has been limited research on PDAs for patients facing treatment decisions for colorectal cancer. We identified only three studies, two of which are low quality, constituting insufficient evidence to make any definitive conclusions on PDA for the treatment of colorectal cancer. There is some indication that these tools are associated with positive outcomes in this population such as increased knowledge and patient satisfaction. Future studies should develop tools that are usable and acceptable to both patients and clinicians, and evaluate these tools for effectiveness in improving decision making for patients facing treatment decisions for colorectal cancer.

Acknowledgements To the Dartmouth College Biomedical Libraries staff, for their support with literature searches.

Contributors All authors have substantial contributions to the conception or design of the work (SJI, HAJ, GE, M-AD, PS), the acquisition, analysis, or interpretation of data for the work (JG, PM, HAJ, SJI), drafting the work or revising it critically for important intellectual content including final approval of the version to be published, and agreement to be accountable for all aspects of the work in ensuring that questions related to the accuracy or integrity of any part of the work are appropriately investigated and resolved (JG, PM, PS, HAJ, MAD, GE, SJI).

Funding The authors have not declared a specific grant for this research from any funding agency in the public, commercial or not-for-profit sectors.

Competing interests M-AD was involved in developing Option Grid decision aids. She receives consulting income from EBSCO Health and may receive royalties in the future. She is also a consultant for ACCESS Community Health Network. GE has edited and published books that provide royalties on sales by the publishers: the books include Shared Decision Making (Oxford University Press) and Groups (Radcliffe Press). He has in the past provided consultancy for organisations, including: (1) Emmi Solutions LLC who developed patient decision support tools; (2) National Quality Forum on the certification of decision support tools; (3) Washington State Health Department on the certification of decision support tools; (4) SciMentum LLC, Amsterdam (workshops for shared decision making). He is the Founder and Director of \&think LLC which owns the registered trademark for Option Grids TM patient decision aids. He is the Founder and Director of SHARPNETWORK LLC, a provider of training for shared decision making. He provides advice in the domain of shared decision making and patient decision aids to: (1) Access Community Health Network, Chicago (Federally Qualified Medical Centers); (2) EBSCO Health Option Grids TM patient decision aids; (3) Bind Insurance, (4) PatientWisdom Inc; (5) abridge Al Inc. GE academic interests are focussed on shared decision making and coproduction. He owns copyright in measures of shared decision making and care integration, namely collaboRATE, integRATE, consideRATE, coopeRATE, toleRATE, Observer OPTION-5 and Observer OPTION12.

Patient consent for publication Not required.

Provenance and peer review Not commissioned; externally peer reviewed.

Data availability statement All data relevant to the study are included in the article or uploaded as supplementary information.

Open access This is an open access article distributed in accordance with the Creative Commons Attribution Non Commercial (CC BY-NC 4.0) license, which permits others to distribute, remix, adapt, build upon this work non-commercially, and license their derivative works on different terms, provided the original work is properly cited, appropriate credit is given, any changes made indicated, and the use is non-commercial. See: http://creativecommons.org/licenses/by-nc/4.0/.

\section{REFERENCES}

1. Fotheringham S, Mozolowski GA, Murray EMA, et al. Challenges and solutions in patient treatment strategies for stage II colon cancer. Gastroenterol Rep 2019;7:151-61.

2. Gray R, Barnwell J, McConkey C, et al. Adjuvant chemotherapy versus observation in patients with colorectal cancer: a randomised study. Lancet 2007;370:2020-9.

3. El Turabi A, Abel GA, Roland M, et al. Variation in reported experience of involvement in cancer treatment decision making: evidence from the National cancer patient experience survey. $\mathrm{Br} \mathrm{J}$ Cancer 2013;109:780-7.

4. Couture J, Chan R, Bouharaoui F. Patient's preferences for adjuvant postoperative chemoradiation therapy in rectal cancer. Dis Colon Rectum 2005;48:2055-60.

5. Neuman HB, Charlson ME, Temple LK. Is there a role for decision AIDS in cancer-related decisions? Crit Rev Oncol Hematol 2007;62:240-50.

6. Gattellari M, Butow PN, Tattersall MH. Sharing decisions in cancer care. Soc Sci Med 2001;52:1865-78.

7. Bruera E, Sweeney C, Calder K, et al. Patient preferences versus physician perceptions of treatment decisions in cancer care. J Clin Oncol 2001;19:2883-5.

8. Thomas R, Thornton H, Mackay J. Patient information materials in oncology: are they needed and do they work? Clin Oncol 1999;11:225-31.

9. Butow PN, Solomon M, Young JM, et al. Consumer impact of an interactive decision aid for rectal cancer patients offered adjuvant therapy. Colorectal Dis 2006;8:676-82.

10. Schroy PC, Emmons KM, Peters E, et al. Aid-Assisted decision making and colorectal cancer screening. Am J Prev Med 2012;43:573-83.

11. Elwyn G. Developing a quality criteria framework for patient decision AIDS: online international Delphi consensus process. BM 2006;333:417-0.

12. Stacey D, Volk R. What are patient decision AIDS? 2017. Available: http://ipdas.ohri.ca/what.html [Accessed 31 Oct 2018].

13. Stacey $\mathrm{D}$, Légaré $\mathrm{F}$, Lewis $\mathrm{K}$, et al. Decision AIDS for people facing health treatment or screening decisions. Cochrane Database Syst Rev 2017;19.

14. Waljee JF, Rogers MAM, Alderman AK. Decision AIDS and breast cancer: do they influence choice for surgery and knowledge of treatment options? J Clin Oncol 2007;25:1067-73.

15. Lin GA, Aaronson DS, Knight SJ, et al. Patient decision AIDS for prostate cancer treatment: a systematic review of the literature. $C A$ Cancer J Clin 2009;59:379-90.

16. Leighl NB, Shepherd FA, Zawisza D, et al. Enhancing treatment decision-making: pilot study of a treatment decision aid in stage IV non-small cell lung cancer. Br J Cancer 2008;98:1769-73.

17. Volk RJ, Linder SK, Lopez-Olivo MA, et al. Patient decision AIDS for colorectal cancer screening: a systematic review and meta-analysis. Am J Prev Med 2016;51:779-91.

18. Ouzzani M, Hammady H, Fedorowicz Z, et al. Rayyan-a web and mobile APP for systematic reviews. Syst Rev 2016;5:210.

19. Higgins JPT, Altman DG, Gøtzsche PC, et al. The Cochrane collaboration's tool for assessing risk of bias in randomised trials. BMJ 2011;343.

20. Downs SH, Black N. The feasibility of creating a checklist for the assessment of the methodological quality both of randomised and non-randomised studies of health care interventions. J Epidemiol Community Health 1998;52:377-84.

21. Vanderbeken I, Kerckhofs E. A systematic review of the effect of physical exercise on cognition in stroke and traumatic brain injury patients. NeuroRehabilitation 2017;40:33-48.

22. Crandall K, Maguire R, Campbell A, et al. Exercise intervention for patients surgically treated for non-small cell lung cancer (NSCLC): a systematic review. Surg Oncol 2014;23:17-30.

23. Leighl NB, Shepherd HL, Butow PN, et al. Supporting treatment decision making in advanced cancer: a randomized trial of a decision aid for patients with advanced colorectal cancer considering chemotherapy. J Clin Oncol 2011;29:2077-84.

24. Wu RC, Boushey RP, Scheer AS, et al. Evaluation of the rectal cancer patient decision aid: a before and after study. Dis Colon Rectum 2016;59:165-72.

25. Miles A, Chronakis I, Fox J, et al. Use of a computerised decision aid $(\mathrm{dA})$ to inform the decision process on adjuvant chemotherapy in patients with stage II colorectal cancer: development and preliminary evaluation. BMJ Open 2017;7:e012935

26. Leighl NB, Butow PN, Clarke SJ. When the goal of treatment is not cure: development of a decision aid for patients with metastatic colorectal cancer. Sunshine Coast, Qld, Australia: Presented at the Medical Oncology Group of Australia Annual General Meeting, 2001: 10-12.

27. Fiset V, O'Connor AM, Evans W, et al. Development and evaluation of a decision aid for patients with stage IV non-small cell lung cancer. Health Expect 2000;3:125-36.

28. Brundage MD, Feldman-Stewart D, Cosby R, et al. Phase I study of a decision aid for patients with locally advanced non-small-cell lung cancer. J Clin Oncol 2001;19:1326-35. 
29. Holmes-Rovner M, Kroll J, Schmitt N, et al. Patient satisfaction with health care decisions: the satisfaction with decision scale. Med Decis Making 1996;16:58-64.

30. O'Connor AM. Validation of a decisional conflict scale. Med Decis Making 1995;15:25-30.

31. Bennett C, Graham ID, Kristjansson E, et al. Validation of a preparation for decision making scale. Patient Educ Couns 2010;78:130-3.

32. Brown ML, Klabunde CN, Cronin KA, et al. Challenges in meeting healthy people 2020 objectives for cancer-related preventive services, National health interview survey, 2008 and 2010. Prev Chronic Dis 2014;11.
33. Beaver K, Campbell M, Craven O, et al. Colorectal cancer patients' attitudes towards involvement in decision making. Health Expect 2009;12:27-37.

34. Sutton PA, Bourdon-Pierre R, Smith C, et al. Evaluating unmet needs in patients undergoing surgery for colorectal cancer: a patient reported outcome measures study. Colorectal Disease 2019;29.

35. Anderson AS, Steele R, Coyle J. Lifestyle issues for colorectal cancer survivors--perceived needs, beliefs and opportunities. Support Care Cancer 2013;21:35-42.

36. Bossema E, Stiggelbout A, Baas-Thijssen M, et al. Patients preferences for low rectal cancer surgery. Eur J Surg Oncol 2008;34:42-8. 